\title{
Multi-Channel Speaker Verification for Single and Multi-talker Speech
}

\author{
Saurabh Kataria ${ }^{1 \dagger *}$, Shi-Xiong Zhang ${ }^{2 \dagger}$, Dong $Y^{2}$ \\ ${ }^{1}$ Center for Language and Speech Processing, Johns Hopkins University, Baltimore, MD, USA \\ ${ }^{2}$ Tencent AI Lab, Bellevue, WA, USA \\ skatarilejh.edu, auszhang@tencent.com, dyu@tencent.com
}

\begin{abstract}
To improve speaker verification in real scenarios with interference speakers, noise, and reverberation, we propose to bring together advancements made in multi-channel speech features. Specifically, we combine spectral, spatial, and directional features, which includes inter-channel phase difference, multichannel sinc convolutions, directional power ratio features, and angle features. To maximally leverage supervised learning, our framework is also equipped with multi-channel speech enhancement and voice activity detection. On all simulated, replayed, and real recordings, we observe large and consistent improvements at various degradation levels. On real recordings of multi-talker speech, we achieve a $36 \%$ relative reduction in equal error rate w.r.t. single-channel baseline. We find the improvements from speaker-dependent directional features more consistent in multi-talker conditions than clean. Lastly, we investigate if the learned multi-channel speaker embedding space can be made more discriminative through a contrastive loss-based fine-tuning. With a simple choice of Triplet loss, we observe a further $8.3 \%$ relative reduction in EER.
\end{abstract}

Index Terms: multi-channel speaker verification, multi-talker, overlapped speech, speech separation, joint learning

\section{Introduction}

Speech devices are getting equipped with multi-channel and multi-modal information, in turn improving spatial ambiguity and directivity [1 2]. Such information is fused at various levels: feature, embedding, or score [3]. This is shown beneficial for Automatic Speech Recognition [4], Speaker Recognition [5], Speech Enhancement [6], and Source Separation [7].

Several spectral, spatial, and directional features [1] are proposed for multi-channel version of such problems. In [8], authors use multi-channel sinc convolution filters and show the importance of phase for time-domain multi-channel speech enhancement. Source Separation work of [9] proposed multichannel Deep Clustering which employs Inter-channel Phase Difference (IPD) and asserts that spatial features are helpful even for arbitrary mic configurations. [6] improved over IPD features through Inter-channel Convolution Difference (ICD) features by directly learning on multi-channel temporal data. In [10], authors showed the effectiveness of Angle Features (AF) [11] for targeted speech extraction.

Multi-Channel Speaker Verification (MCSV) is an underexplored problem with no common benchmarks except CHiME-5 [12]. It is pursued mostly using pre-processing techniques like dereverberation and mask-based beamforming. In [13], authors combined Weighted Prediction Error (WPE) dereverberation and Minimum Variance Distortion-less Response (MVDR) beamforming for MCSV. In [14], authors search for

*This work was done while Saurabh Kataria was a research intern at Tencent AI Lab, USA. †These authors contributed equally. optimal beamformer among variants of Ideal Ratio Mask (IRM) based MVDR and Generalized Eigen-Value (GEV) beamformers. [15] showed the utility of multi-channel speech enhancement for MCSV. [5] found that multi-channel Time-Frequency (T-F) representations are superior to single-channel especially when 3D convolutions are used instead of 2D in a deep multichannel input based Convolutional Neural Network (CNN).

We believe prior works are not comprehensive in terms of leveraging all available information. For example, they do not investigate if spatial and location clues of sound sources can help speaker verification. [9] noted that linear beamformer filtering cannot capture multi-channel non-linear information. Towards building a complete multi-channel speaker embedding system, we propose to incorporate various multi-channel features and learn a multi-channel $\mathrm{CNN}$ embedding network in conjunction with joint (multi-channel) enhancement and Voice Activity Detection (VAD). Specifically, we pursue textindependent wide-band informed [16] (opposed to blind [17]) MCSV.

Our contributions are: (1) we provide the first study to compare and combine various multi-channel spectral, spatial, and directional speech features for robust MCSV, and report consistent improvements on real far-field data; (2) we devise a comprehensive location-aware speaker verification setup with joint enhancement, VAD, and realistic single and multi-talker test scenarios; (3) we demonstrate that this multi-channel system performance can be improved via contrastive fine-tuning.

\section{Multi-Channel Speaker Verification}

\subsection{Proposed Multi-Task Supervised Learning Framework}

To maximally exploit supervised learning, we devise a multitask framework that jointly optimizes three multi-channel tasks: speaker embedding learning, voice activity detection, and speech enhancement. Thanks to simulation, we utilize ground truth labels for the latter two tasks. Using the initial singlechannel clean speech, we compute its energy VAD and the spectrogram as targets for the two tasks respectively. Loss for complete framework is $\mathcal{L}_{\text {all }}=\mathcal{L}_{\text {BASE }}+\lambda_{\text {enh }} \mathcal{L}_{\text {enh }}$, where $\mathcal{L}_{\mathrm{BASE}}=\mathcal{L}_{\mathrm{emb}}+\lambda_{\mathrm{VAD}} \mathcal{L}_{\mathrm{VAD}}$, and $\lambda_{\mathrm{VAD}}, \lambda_{\text {enh }}$ are regularization weights for VAD and enhancement task respectively. They are set to 0.1 and 0.0005 . $\mathcal{L}_{\text {emb }}$ is Cross Entropy (CE) loss, $\mathcal{L}_{\text {VAD }}$ is frame-wise Binary Cross Entropy (BCE) loss, $\mathcal{L}_{\text {enh }}$ is spectrogram-domain Mean Absolute Error (MAE) loss, and $\mathcal{L}_{\text {BASE }}$ is the loss for the BASE model which is a single-channel joint speaker embedding and VAD system without the speech enhancement module.

Fig 1 illustrates our proposal. Note that the input features for the three tasks are identical but their outputs are combined later. Our methodology is to incrementally incorporate various multi-channel features on top of the BASE system in order to maximize speaker recognition performance. We do not investi- 
gate feature fusion schemes and simply concatenate all features along the channel dimension of the input layer of $\mathrm{CNN}$. We hope to observe the complementary effect of such features [9] since some of them may under-perform in adverse scenarios. For e.g., under heavy reverberations, IPDs get degraded. Hence, we are interested in a MCSV system which is robust and generalizes well. To explore this, we choose a Mandarin test set that is language-mismatched with the train set (English) and is corrupt with reverberations, background noises, and speaker interferences. Since there is no MCSV work directly comparable to our setup, for the baseline, we use the standard single-channel 80-D Log-Mel Filter Bank (LMFB) features (BASE system).

\subsection{Large-Margin Contrastive Fine-Tuning}

We investigate if the learned multi-channel speaker embedding space can be made more discriminative using contrastive lossbased post-processing. We are inspired by [18], which proposed to switch from CE loss to Triplet loss towards the end of speaker embedding training for superior generalization. On the pre-trained multi-channel model, we minimize $\mathcal{L}_{\text {triplet }}=$ $f_{\beta}(d(a, p)-d(a, n)+m)$, where $f_{\beta}(x)=\beta^{-1} \log (1+$ $\exp (\beta x)), \mathcal{L}_{\text {triplet }}$ refers to the contrastive (Triplet) loss, $f_{\beta}(\cdot)$ is the softplus function (smooth version of hinge function [19]), $\beta$ is a non-negative constant, and $d$ is the Euclidean distance function. In the Triplet loss terminology [18], $a$ is an anchor example, $p$ is a positive example, $n$ is a negative example, and $m$ is the margin. $a$ and $p$ belong to the same class while $n$ belongs to a different class. For triplet formation, we follow the hardest mining strategy [19]. It refers to choosing hardest positive and hardest negative example for each anchor with $d$ as the criterion. For this, the training batch must contain multiple examples per speaker. We follow $P K$ sampling i.e. choose $K$ examples per $P$ unique speakers. We are particularly interested in large margins in this formulation.

\section{Multi-Channel Features}

Inter-Channel Phase Difference: IPD is a common spatial feature which measures the phase difference between complex Short-Time Fourier Transform (STFT) of signals at two different microphones.

$$
\operatorname{IPD}_{(i, j), t f}=\angle\left(\frac{Y_{i, t f}}{Y_{j, t f}}\right), i, j=1 \ldots M, j \neq i .
$$

Here, $M$ is the number of microphones in the array, $i, j$ are microphone indices, $(t, f)$ is the current T-F bin, and $Y$ is the STFT. Note that this gives us $M(M-1)$ pairs but we later choose only a pre-defined small subset. Cosine and sine of IPD are extensively used in prior works [6, 7, 9] and they are referred to as cosIPD and sinIPD.

Directional Power Ratio (DPR): We adopt directional features from [20] based on the output power of multi-look fixed beamformers and direction beam of target speaker $\theta_{p}$. For a set of direction grid of beams $\left\{\theta_{1}, \ldots, \theta_{P}\right\}$ and the corresponding filters $\mathbf{w}_{f}\left(\theta_{p}\right)$,

$$
\operatorname{DPR}_{t f}\left(\theta_{p}\right)=\frac{\left\|\mathbf{w}_{f}^{\mathrm{H}}\left(\theta_{p}\right) \mathbf{Y}_{t f}\right\|_{2}^{2}}{\sum_{p=1}^{P}\left\|\mathbf{w}_{f}^{\mathrm{H}}\left(\theta_{p}\right) \mathbf{Y}_{t f}\right\|_{2}^{2}},
$$

where $\mathbf{Y}$ is the mixed multi-channel complex spectrum. The DPR features quantify how well a T-F bin is represented by a signal from a direction beam $\theta_{p}$.
Angle Features: We adopt the Angle Features (AF) from [2]. The AF is formed according to the angle $\theta$ of a target speaker and measures the cosine distance between the steering vector and IPD:

$$
\begin{aligned}
A_{t f}\left(\theta_{t}\right) & =\sum_{(i, j)}\left\langle\mathbf{e}^{\operatorname{TPD}(i, j), t f}, \mathbf{e}^{\operatorname{IPD}(i, j), t f}\right\rangle \\
\operatorname{TPD}_{(i, j), t f} & =2 \pi \Delta_{i j} \cos \left(\theta_{t}\right) f /\left(c \cdot f_{s}\right)
\end{aligned}
$$

Vector $\mathbf{e}^{(\cdot)}=[\cos (\cdot), \sin (\cdot)]^{\top}, \operatorname{TPD}_{(i, j), t f}$ (Target-dependent Phase Difference) is the phase delay between microphones $i$ and $j$ for a plane wave with frequency $f$. The plane wave is travelling from an angle $\theta_{t}$ (target speaker's angle at time $t$ ), $\Delta_{i j}$ is the distance between the $i, j$ microphone pair, $c$ is the sound velocity and $f_{s}$ is sampling rate. In the training stage, the angle of target speaker $\theta$ is known as multi-channel audios were generated by simulation. In practice, the angle of the target speaker $\theta_{t}$ can be estimated by the face tracking system [21] or audiobased source localization [22]. Details of angle estimation are beyond the scope of this work and were discussed in [23].

Multi-Channel SincNet Features: A sinc convolution layer [24] learns data-dependent 1-D filters. We extend such layer to multi-channel input as done in multi-channel speech enhancement work of [8]. We learn $C_{\text {sinc }}=257$ filters so that the resultant feature dimension is compatible with other features. $i$ th sinc filter $\mathbf{s}_{i, t}$ is windowed and subsequently convolved with time-domain signal of every channel and given by:

$$
\mathbf{s}_{i, t}=2 f_{i, \text { low }} \operatorname{sinc}\left(2 \pi f_{i, \text { low }} t\right)-2 f_{i, \text { high }} \operatorname{sinc}\left(2 \pi f_{i, \text { high }} t\right)
$$

$f_{i, \text { low }}$ and $f_{i, \text { high }}$ are learnable low and high cutoff frequency parameters [8] and $t$ is the temporal index. We propose to use the resultant features, MultChanSinc, as an alternative to LPS+IPD since it combines spectral and spatial information. For fair comparison, we provide MultChanSinc same channel information as the IPD.

\section{Experiments}

Our study is device-specific and is performed with a 15-channel non-uniform linear microphone array with spacing 7-6-5-4-3-21-1-2-3-4-5-6-7 in $\mathrm{cm}$ and a 180-degree camera. The proposed multi-channel speaker verification system was evaluated under four far-field conditions - 1) simulated single-talker speech, 2) simulated multi-talker speech, 3) replayed recordings of multitalker speech, and 4) real recording of multi-talker speech.

For simulated single and multi-talker setups, test data is originally single-channel $16 \mathrm{KHz}$ and is simulated to $15-$ channel. The details of the simulation can be found in the Alg. 1 of [2]. The evaluation data consists of clean internal Mandarin corpus ( $36 \mathrm{hrs}, 364$ speakers). For data augmentation, we use a large internal noise and Room Impulse Response (RIR) corpus which contains 700 noise files and 3000 RIRs. The RIRs are multi-channel signals simulated in various virtual room configurations. They are also similarly split to create disjoint training, validation, and evaluation data. We constrain all sound sources (target speaker, interference speaker, noise sources) to the azimuth angle range of $\left(0^{\circ}, 180^{\circ}\right)$. For DPR features, we choose the spatial resolution of $10^{\circ}$ and hence $P=10$ in Eq. 2. Ignoring spatial ambiguity issue [6], we keep the angle between the target and interference speaker unconstrained. The position of all sources is static. Only one interference speaker is allowed with probability $p_{\text {tar }}$. For training and validation, Signalto-Noise Ratios (SNRs) $\in\{-3,0,6,12,18\} \mathrm{dB}$, Signal-toInterference Ratios (SIRs) $\in\{0,3,6\} \mathrm{dB}$, and $p_{\text {tar }}=0.15$. For 


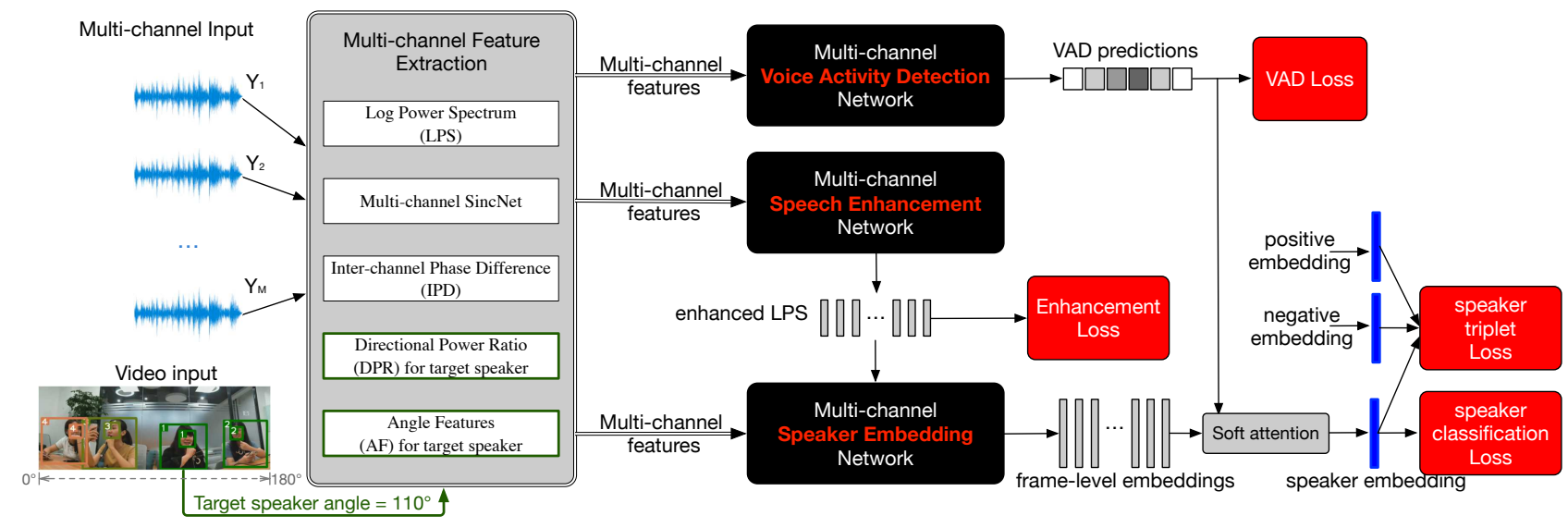

Figure 1: Schematic of the proposed multi-channel speaker verification based on multi-channel features. In practice, face and body detection in video can provide locations of each speaker but not who is speaking or when.

evaluation on simulated data, we create 10,000 one-vs-one trials with equal number of target and non-target trials, with SNRs $\in\{-2,2,4,8,10,14\} \mathrm{dB}$, SIRs $\in\{2,4\} \mathrm{dB}$, and $p_{\text {tar }}=0.2$.

For the replayed multi-talker test set, 2500 (1h) utterances from 364 speakers are recorded in a $10 \times 5 \times 3 \mathrm{~m}^{3}$ meeting room. As shown in Fig. 2 (a), two loudspeakers are used to replay different utterances of the internal Mandarin corpus simultaneously to generate overlapped speech. We create 5,000 one-vsone trials with an equal number of target and non-target trials for replayed data. For the real recording of the multi-talker test set, $10 \mathrm{~h}$ partially overlapped speech from 50 speakers in 100 debate meetings are recorded in several meeting rooms. Each speaker has recorded 3-5 single-talker utterances using the same multichannel device, as enrollment. We create another 5,000 onevs-one trials from segmented real recording data. This multichannel audio-visual data of real debate meetings will be released in the near future [23].

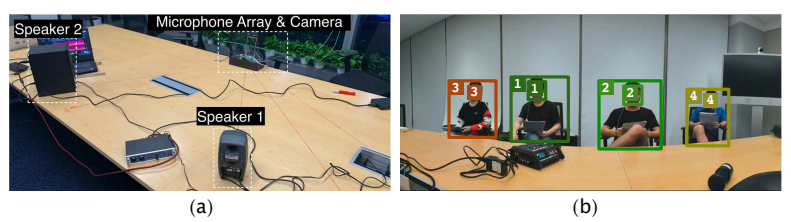

Figure 2: (a) Replayed recording of multi-talker speech. (b) Real recording of multi-talker speech with 15-channel microphone array and 180-degree camera.

To construct training data, we first combine a portion of VoxCeleb [25] (450 hrs, 7285 speakers) and a small amount of internal Mandarin data (50 hrs, 2265 speakers). This gives us approximately $500 \mathrm{hrs}$ and 9550 speakers which is then split in 9:1 for training and validation data. All STFT based features are extracted with 512-point Discrete Fourier Transform (DFT), $25 \mathrm{~ms}$ analysis window, and $10 \mathrm{~ms}$ shift. For IPD computation, we experiment with two sets of mic pairs:

v0: $(0,7),(2,7),(3,11),(5,9),(11,5),(9,3)$

v1: $(0,2),(3,5),(6,8),(9,11),(12,14),(1,4),(5,8),(9,12)$

When features like DPR and AF use target speaker location only, they are referred to as " $\mathrm{DPR}(1)$ " and " $\mathrm{AF}(1)$ ". When interference speaker location is also used, we term them "DPR(2)" and " $\mathrm{AF}(2)$ ". When interference speaker is absent, interference noise source location is used instead.

For the speaker embedding network, we use the standard ResNet-34 architecture with Time-Average Pooling (TAP) of embeddings [26]. For the speech enhancement network, we use the multi-channel TasNet with 4 Temporal Convolutional Network (TCN) blocks. Each TCN block is stacked by 8 Dilated 1-D ConvBlock with exponentially increased dilation factors $2^{0}, 2^{1}, \ldots, 2^{7}$. Each dilated 1-D ConvBlock consists of a $1 \times 1$ convolutional layer, a depth-wise separable convolution layer (D-Conv) [27], with PReLU [28] activation function, and normalization added between each two convolution layers. Skip connection is added in each dilated 1-D ConvBlock. The early fusion method [29] was adopted to combine LPS, IPD, and AF features before feeding into a TCN block. For VAD network, we use a 2-layer Bi-Directional Long Short Term Memory (BLSTM). We do Adam optimization with initial Learning Rate (LR) of 0.0075 , batch size of 240,8 epochs, and a simple LR scheduler (decrease by $50 \%$ when validation loss does not improve). For contrastive fine-tuning experiments, $K=4$ for $P K$ sampling, $P=60$ (since batch size is 240 ), $\beta=1$ for softplus function, and LR is 0.01 with similar scheduling as before. Our 512-D speaker embeddings (extracted from the penultimate layer of ResNet) are compared using a simple cosine similarity backend [18] and results are reported in terms of validation accuracy, Equal Error Rate (EER), and minimum Decision Cost Function (minDCF) with the target prior probability of 0.05 . The lower the EER and minDCF, the better.

\section{Results}

5.1. The benefit of multi-channel features over singlechannel features for Speaker Verification

In Table 1 we evaluate our multi-channel system on four testing scenarios. First, we observe that 257-D Log Power Spectrum (LPS) features are better than the baseline 80-D LMFBs perhaps due to higher dimensionality. In clean conditions, MultChanSinc features are better than single-channel features but perform worse or equivalent to LPS in noisy conditions. This suggests that these features might not be robust to noise or are sensitive to its hyper-parameters. LPS+cosIPD are conventional choice of features for MCSV and we find them superior to MultChanSinc in our case. By experimenting with two choices of microphone pairs, we observe that this choice is important and perhaps can even be learned. We find v0 to be much superior to $\mathrm{v} 1$ even though the latter is a bigger set. They also achieve the best clean condition EER. Adding beamformer features (DPR) hurt in clean condition while roughly maintaining performance in other cases. This is possibly because these features fail to complement other features under our simple feature 
Table 1: The results of multi-channel systems on four test sets, including simulated far-field single talker speech, simulated multi-talker speech, replayed recording of multi-talker speech, real recording of multi-talker speech. "-" marks clean testing conditions when $D P R(2), A F(2)$ cannot be computed due to non-existence of inteference sources. Enh and VAD indicate if enhancement and VAD module are present respectively. val acc (in \%) is the corresponding system validation accuracy. "DPR(2)" and "AF(2)" means the locations of both target and interference speaker are used.

\begin{tabular}{|c|c|c|c|c|c|c|c|c|c|c|c|}
\hline & & & & \multicolumn{2}{|c|}{ simulated (single) } & \multicolumn{2}{|c|}{ simulated (multi-talker) } & \multicolumn{2}{|c|}{ replay (multi-talker) } & \multicolumn{2}{|c|}{ real (multi-talker) } \\
\hline Input Features & Enh & VAD & val Acc & EER $(\%)$ & $\operatorname{minDCF}$ & $\operatorname{EER}(\%)$ & $\operatorname{minDCF}$ & EER (\%) & $\operatorname{minDCF}$ & $\operatorname{EER}(\%)$ & $\operatorname{minDCF}$ \\
\hline single channel LMFB & $\bar{x}$ & $\overline{\sqrt{ }}$ & $\overline{62.3}$ & $\overline{13.9}$ & $\overline{0.747}$ & $\overline{24.9}$ & $\overline{0.921}$ & $\overline{28.4}$ & 0.968 & $\overline{\overline{19.3}}$ & $\overline{0.848}$ \\
\hline single channel LPS & $x$ & $\sqrt{ }$ & 64.0 & 12.7 & 0.660 & 21.5 & 0.875 & 25.0 & 0.952 & 18.6 & 0.839 \\
\hline MultChanSinc & $x$ & $\sqrt{ }$ & 66.6 & 12.1 & 0.654 & 23.0 & 0.902 & 26.6 & 0.917 & 17.7 & 0.834 \\
\hline$\overline{\mathrm{LPS}}+\cos$ IPDv0 & $x$ & $\bar{J}$ & 66.4 & $\mathbf{1 0 . 5}$ & 0.645 & 19.9 & 0.840 & 22.2 & 0.884 & 15.2 & 0.784 \\
\hline LPS + cosIPDv1 & $x$ & $\checkmark$ & 67.2 & 15.2 & 0.713 & 23.9 & 0.846 & 25.9 & 0.887 & 17.4 & 0.831 \\
\hline LPS + cosIPDv0 + DPR(1) & $x$ & $\sqrt{ }$ & 69.1 & 13.7 & 0.755 & 20.0 & 0.900 & 22.1 & 0.933 & 15.0 & 0.752 \\
\hline $\mathrm{LPS}+\cos \mathrm{IPDv} 0+\mathrm{AF}(1)$ & $x$ & $\sqrt{ }$ & 71.0 & 14.2 & 0.740 & 19.1 & 0.818 & 20.7 & 0.882 & 14.5 & 0.746 \\
\hline $\mathrm{LPS}+\cos I \mathrm{PDv} 0+\mathrm{DPR}(1)+\mathrm{AF}(1)$ & $x$ & $\sqrt{ }$ & 70.9 & 12.0 & 0.658 & 16.9 & 0.818 & 18.1 & 0.866 & 14.1 & 0.725 \\
\hline LPS + cosIPDv0 + DPR $(1)+\mathrm{AF}(1)$ & $\sqrt{ }$ & $\checkmark$ & 70.6 & 10.5 & 0.611 & 16.4 & $\mathbf{0 . 8 0 7}$ & 17.7 & 0.860 & 12.9 & 0.717 \\
\hline $\mathrm{LPS}+\cos \mathrm{IPDv} 0+\mathrm{DPR}(2)+\mathrm{AF}(2)$ & $\sqrt{ }$ & $\sqrt{ }$ & 71.7 & - & - & $\overline{16.3}$ & 0.812 & $\overline{17.5}$ & $\overline{0.858}$ & 12.6 & 0.715 \\
\hline LPS + cosIPDv0 + DPR $(2)+\mathrm{AF}(2)$ & $\checkmark$ & $x$ & 73.5 & - & - & 17.4 & 0.822 & 18.3 & 0.862 & 14.2 & 0.739 \\
\hline
\end{tabular}

Table 2: The detailed results of simulated multi-talker speech on different SIRs and SNRs.

\begin{tabular}{|c|c|c|c|c|c|c|c|c|}
\hline \multirow{2}{*}{ Input Features } & \multirow{2}{*}{ Enh } & \multirow{2}{*}{ VAD } & & \multicolumn{2}{|c|}{ SIR $=0$, SNR $=$} & \multicolumn{3}{|c|}{ SIR=6, SNR= } \\
\cline { 5 - 10 } & & & -2 & 5 & 15 & -2 & 5 & 15 \\
\hline single channel LMFB & $\boldsymbol{x}$ & $\checkmark$ & 32.0 & 24.6 & 20.6 & 30.8 & 22.8 & 18.1 \\
\hline single channel LPS & $\boldsymbol{x}$ & $\checkmark$ & 27.4 & 21.6 & 18.0 & 26.2 & 20.1 & 16.7 \\
\hline LPS+cosIPD & $\boldsymbol{X}$ & $\checkmark$ & 26.1 & 19.9 & 16.1 & 24.9 & 18.4 & 14.5 \\
\hline LPS+cosIPD+DPR(1)+AF(1) & $\boldsymbol{x}$ & $\checkmark$ & 23.8 & 17.1 & 15.8 & 22.1 & 17.3 & 15.4 \\
\hline LPS+cosIPD+DPR(1)+AF(1) & $\checkmark$ & $\checkmark$ & $\mathbf{1 9 . 7}$ & $\mathbf{1 6 . 5}$ & $\mathbf{1 4 . 9}$ & $\mathbf{1 9 . 2}$ & $\mathbf{1 6 . 2}$ & $\mathbf{1 4 . 1}$ \\
\hline
\end{tabular}

fusion scheme. The trend is similar for Angle Features (AF) although they seem superior to DPR features. When both types of directional features are combined, we observe complementary behavior and non-target cancellation ability. When speech enhancement is introduced, we observe consistent and significant gains. Using interference source location gives us the best system which outperforms in almost all noisy conditions. We expect this improvement to be higher when the number of sources is more than (current) two. We also observed that sinIPD features do not deliver more information to the system (not listed here). Finally, from the ablation experiment (last row), we can see the importance of VAD in our joint learning system.

A critical observation is that progressively incorporating more information in our methodology leads to consistent improvement in validation accuracy, i.e. better speaker identification accuracy, but the generalization performances measured by speaker verification tasks vary. Finally, we note that combining the LPS + cosIPDv0 + DPR + AF features has almost closed the performance gap between the real recording of multi-talker speech (12.6\%) and single-talker speech (10.5\%).

\subsection{Multi-channel system evaluation on various SNR, SIR}

In Table 2 we evaluate few key feature combinations on specific (SNR, SIR) pairs. We note that the trend of consistent improvement with the addition of features holds true especially in challenging conditions (low SNR, low SIR). On one cleaner condition (SIR=6, SNR=15), directional features are worse than spatial features. This suggests the sub-optimality of our feature concatenation scheme for directional features. We also observe that the performance of multi-channel features is always better than single-channel and even in high SNR, high SIR conditions. Combining spectral, spatial, and directional features in low $\mathrm{SNR}=-2 \mathrm{~dB}$ gives almost identical performance for both $\mathrm{SIR}=0$ and $\mathrm{SIR}=6$. This demonstrates the strong ability of our system to handle interference speakers.

\subsection{Contrastive fine-tuning of the multi-channel system}

We also investigate if one of our best multi-channel systems in Table 1 (LPS + cosIPDv0 + DPR(1) + AF(1)) can be finetuned to improve verification performance by a simple choice of contrastive loss: Triplet Loss. By experimenting with various values of margin $m$, we obtain large improvements in clean as well as noisy conditions. This suggests that softmax training leaves some scope of improvement in embedding space. $m=2$ gives the best results contrary to small values like 0.1 and 0.2 used in previous works [18, 30]. For simulated single and multitalker tests, compared to the single-channel baseline of Table 1 we reduce EER from $16.9 \%$ to $15.5 \%$. Hence, we demonstrate that MCSV performance can be improved via a better design of input feature space as well as target embedding space.

Table 3: Fine-tuning the multi-channel system, $L P S+\operatorname{cosIPDvO}$ $+D P R(1)+A F(1)$ with VAD, using different Triplet margin $m$.

\begin{tabular}{|c|c|c|c|c|}
\cline { 2 - 5 } \multicolumn{1}{c|}{} & \multicolumn{2}{c|}{ simulated (single-talker) } & \multicolumn{2}{c|}{ simulated (multi-talker) } \\
\cline { 2 - 5 } \multicolumn{1}{c|}{} & EER (\%) & minDCF & EER (\%) & minDCF \\
\hline no fine-tuning & 12.0 & $\mathbf{0 . 6 5 8}$ & 16.9 & 0.818 \\
\hline$m=0.3$ & 11.0 & 0.705 & 16.8 & 0.813 \\
\hline$m=1$ & $\mathbf{1 0 . 2}$ & 0.697 & 16.4 & 0.834 \\
\hline $\mathbf{m}=\mathbf{2}$ & 10.5 & 0.680 & $\mathbf{1 5 . 5}$ & $\mathbf{0 . 7 8 0}$ \\
\hline$m=3$ & 11.3 & 0.693 & 16.4 & 0.827 \\
\hline
\end{tabular}

\section{Conclusion}

To advance robust location-aware multi-channel Speaker Verification, we tackle the problem of designing better input features as well as embedding space. For the former task, we explored various combinations of spectral, spatial, and directional features to find that single-channel baseline can be vastly improved under all testing conditions with combinations of spectral and spatial features. We find the benefit of adding directional features more prominent in multi-talker conditions while an analysis showed that further exploration is required to improve our simple feature concatenation scheme. Overall, we observe $36 \%$ relative reduction in Equal Error Rate (EER) on real recordings. We also show that the discriminativity of speaker embedding space can be significantly improved via a contrastive loss-based fine-tuning of our multi-channel system. In the future, we can investigate (1) feature fusion learning schemes; (2) robustness to sound source(s) location information (modality robustness problem [2]); and (3) explore more multi-channel features like Inter-channel Convolution Differences (ICD) [6]. 


\section{References}

[1] Z. Chen, X. Xiao, T. Yoshioka, H. Erdogan, J. Li, and Y. Gong, "Multi-channel overlapped speech recognition with location guided speech extraction network," in 2018 IEEE Spoken Language Technology Workshop (SLT). IEEE, 2018, pp. 558565.

[2] R. Gu, S.-X. Zhang, Y. Xu, L. Chen, Y. Zou, and D. Yu, "Multimodal multi-channel target speech separation," IEEE Journal of Selected Topics in Signal Processing, 2020.

[3] L. MOŠNER, "Far-field speaker verification incorporating multichannel processing," BRNO UNIVERSITY OF TECHNOLOGY thesis, 2020.

[4] T. N. Sainath, R. J. Weiss, K. W. Wilson, B. Li, A. Narayanan, E. Variani, M. Bacchiani, I. Shafran, A. Senior, K. Chin et al., "Multichannel signal processing with deep neural networks for automatic speech recognition," IEEE/ACM Transactions on Audio, Speech, and Language Processing, vol. 25, no. 5, pp. 965979, 2017.

[5] D. Cai, X. Qin, and M. Li, "Multi-channel training for end-to-end speaker recognition under reverberant and noisy environment." in INTERSPEECH, 2019, pp. 4365-4369.

[6] R. Gu, S.-X. Zhang, L. Chen, Y. Xu, M. Yu, D. Su, Y. Zou, and D. Yu, "Enhancing end-to-end multi-channel speech separation via spatial feature learning," in ICASSP 2020-2020 IEEE International Conference on Acoustics, Speech and Signal Processing (ICASSP). IEEE, 2020, pp. 7319-7323.

[7] Z.-Q. Wang and D. Wang, "Combining spectral and spatial features for deep learning based blind speaker separation,' IEEE/ACM Transactions on Audio, Speech, and Language Processing, vol. 27, no. 2, pp. 457-468, 2018.

[8] C.-L. Liu, S.-W. Fu, Y.-J. Li, J.-W. Huang, H.-M. Wang, and Y. Tsao, "Multichannel speech enhancement by raw waveformmapping using fully convolutional networks," IEEE/ACM Transactions on Audio, Speech, and Language Processing, vol. 28, pp 1888-1900, 2020.

[9] Z.-Q. Wang, J. Le Roux, and J. R. Hershey, "Multi-channel deep clustering: Discriminative spectral and spatial embeddings for speaker-independent speech separation," in 2018 IEEE International Conference on Acoustics, Speech and Signal Processing (ICASSP). IEEE, 2018, pp. 1-5.

[10] A. S. Subramanian, C. Weng, M. Yu, S.-X. Zhang, Y. Xu, S. Watanabe, and D. Yu, "Far-field location guided target speech extraction using end-to-end speech recognition objectives," in ICASSP 2020-2020 IEEE International Conference on Acoustics, Speech and Signal Processing (ICASSP). IEEE, 2020, pp. 72997303.

[11] Z.-Q. Wang and D. Wang, "On spatial features for supervised speech separation and its application to beamforming and robust asr," in 2018 IEEE International Conference on Acoustics, Speech and Signal Processing (ICASSP). IEEE, 2018, pp. 5709-5713.

[12] D. Garcia-Romero, D. Snyder, S. Watanabe, G. Sell, A. McCree, D. Povey, and S. Khudanpur, "Speaker recognition benchmark using the chime-5 corpus." in INTERSPEECH, 2019, pp. 15061510

[13] J.-Y. Yang and J.-H. Chang, "Joint optimization of neural acoustic beamforming and dereverberation with $\mathrm{X}$-vectors for robust speaker verification." in INTERSPEECH, 2019, pp. 4075-4079.

[14] H. Taherian, Z.-Q. Wang, and D. Wang, "Deep learning based multi-channel speaker recognition in noisy and reverberant environments," in Interspeech, 2019.

[15] H. Taherian, Z.-Q. Wang, J. Chang, and D. Wang, "Robust speaker recognition based on single-channel and multi-channel speech enhancement," IEEE/ACM Transactions on Audio, Speech, and Language Processing, vol. 28, pp. 1293-1302, 2020.

[16] M. Delcroix, K. Zmolikova, K. Kinoshita, A. Ogawa, and T. Nakatani, "Single channel target speaker extraction and recognition with speaker beam," in 2018 IEEE International Conference on Acoustics, Speech and Signal Processing (ICASSP). IEEE, 2018, pp. 5554-5558.
[17] D. Yu, M. Kolbæk, Z.-H. Tan, and J. Jensen, "Permutation invariant training of deep models for speaker-independent multi-talker speech separation," in 2017 IEEE International Conference on Acoustics, Speech and Signal Processing (ICASSP). IEEE, 2017, pp. 241-245.

[18] C. Li, X. Ma, B. Jiang, X. Li, X. Zhang, X. Liu, Y. Cao, A. Kannan, and Z. Zhu, "Deep speaker: an end-to-end neural speaker embedding system," arXiv preprint arXiv:1705.02304, vol. 650, 2017.

[19] A. Hermans, L. Beyer, and B. Leibe, "In defense of the triplet loss for person re-identification," arXiv preprint arXiv:1703.07737, 2017.

[20] R. Gu, L. Chen, S.-X. Zhang, J. Zheng, Y. Xu, M. Yu, D. Su, Y. Zou, and D. Yu, "Neural spatial filter: Target speaker speech separation assisted with directional information," in Proc. Interspeech, 2019.

[21] D. E. King, "Dlib-ml: A machine learning toolkit," Journal of Machine Learning Research, vol. 10, pp. 1755-1758, 2009.

[22] A. S. S. et. al., "Directional ASR: A new paradigm for E2E multispeaker speech recognition with source localization," in ICASSP. IEEE, submitted.

[23] S.-X. Zhang, Y. Xu, M. Yu, J. Yu, M. Jin, D. Su, and D. Yu, "M33. Multi-Modal Multi-channel dataset for cocktail party problems," to be released, 2021

[24] M. Ravanelli and Y. Bengio, "Speaker recognition from raw waveform with sincnet," in 2018 IEEE Spoken Language Technology Workshop (SLT). IEEE, 2018, pp. 1021-1028.

[25] A. Nagrani, J. S. Chung, W. Xie, and A. Zisserman, "Voxceleb: Large-scale speaker verification in the wild," Computer Speech \& Language, vol. 60, p. 101027, 2020.

[26] Y. Jung, Y. Choi, and H. Kim, "Self-adaptive soft voice activity detection using deep neural networks for robust speaker verification," in 2019 IEEE Automatic Speech Recognition and Understanding Workshop (ASRU). IEEE, 2019, pp. 365-372.

[27] F. Chollet, "Xception: Deep learning with depthwise separable convolutions," in Proceedings of the IEEE conference on computer vision and pattern recognition, 2017, pp. 1251-1258.

[28] K. He, X. Zhang, S. Ren, and J. Sun, "Delving deep into rectifiers: Surpassing human-level performance on imagenet classification," in Proceedings of the IEEE international conference on computer vision, 2015, pp. 1026-1034.

[29] R. Gu, J. Wu, S.-X. Zhang, L. Chen, Y. Xu, M. Yu, D. Su, Y. Zou, and D. Yu, "End-to-end multi-channel speech separation," arXiv, pp. arXiv-1905, 2019.

[30] H. Bredin, "Tristounet: triplet loss for speaker turn embedding," in 2017 IEEE international conference on acoustics, speech and signal processing (ICASSP). IEEE, 2017, pp. 5430-5434. 
This figure "replay.jpg.png" is available in "png" format from: http://arxiv.org/ps/2010.12692v2 